\title{
Circular RNA hsa_circ_0043278 inhibits breast cancer progression via the miR-455-3p/ El24 signalling pathway
}

Yue Shi' and Chong Liur

\begin{abstract}
Background: Breast cancer (BC) is one of the major malignancies worldwide. Circular ribonucleic acids (circRNAs) are a class of conserved ribonucleic acid (RNA) molecules that play important roles in various diseases. Recently, circRNAs have been suggested to have diagnostic value and may function as potential diagnostic biomarkers for BC. Previously, hsa_circ_0043278 was found to be downregulated in human BC. However, its role in human BC has not yet been identified.
\end{abstract}

Methods: The levels of hsa_circ_0043278 in BC cell lines were verified by quantitative reverse transcription-polymerase chain reaction (qRT-PCR). The overexpression vector and short hairpin RNA (shRNA) of hsa_circ_0043278 were transfected into MDA-MB-231 and MCF-7 cells, respectively. The effects of hsa_circ_0043278 on tumour cell growth, migration and invasion were measured by 3-(4,5-Dimethylthiazol-2-yl)-2,5-diphenyl-tetrazolium bromide (MTT), colony formation, wound healing and Transwell assays in vitro. A xenograft experiment was conducted to validate the inhibitory effect of hsa_circ_0043278 on tumour growth. The interaction between hsa_circ_0043278 and miR-455-3p was confirmed by a dual-luciferase reporter assay. Mimics and inhibitors of miR-455-3p were designed to confirm the influence of hsa_circ_0043278 on the hsa_circ_0043278/miR-455-3p/etoposide-induced gene 24 (EI24) axis.

Results: Hsa_circ_0043278 was downregulated in BC cell lines. Furthermore, overexpression of hsa_circ_0043278 notably decreased $B C$ cell viability and inhibited $B C$ cell migration and invasion in vitro and suppressed tumour growth in vivo. Downregulation of hsa_circ_0043278 led to the opposite results. Hsa_circ_0043278 expression was negatively correlated with that of miR-455-3p. In addition, mechanistic investigation proved that hsa_circ_0043278 directly bound to miR-455-3p and regulated El24 and NF-kB expression in BC cells.

Conclusion: Hsa_circ_0043278 acts as a tumour suppressor gene in BC through the hsa_circ_0043278/miR-455-3p/ El24 axis and may be regarded as a new prognostic predictor or potential therapeutic target in BC.

Keywords: Breast cancer, Circular RNA, hsa_circ_0043278

\section{Background}

Breast cancer $(\mathrm{BC})$ is the second most common cancer worldwide and is a major cause of female death $[1,2]$. Some classical biomarkers, such as CEA and CA 15-3,

\footnotetext{
*Correspondence: liuchong@cmu.edu.cn

${ }^{2}$ Department of Breast Surgery, The First Affiliated Hospital of China

Medical University, Shenyang 110001, China

Full list of author information is available at the end of the article
}

detected in serum or nipple discharge, are associated with the development of $\mathrm{BC}[3,4]$. But their tumour specificity is unsatisfactory. Furthermore, studies show that several genetic variants are potentially associated with $\mathrm{BC}$ risk, which provides insights into new potential biomarkers for $\mathrm{BC}[5,6]$. However, biomarkers for the diagnosis and prognosis of human $\mathrm{BC}$ remain limited [7]. Hence, it is still important to understand the underlying 
molecular processes of $\mathrm{BC}$ to explore novel biomarkers for $\mathrm{BC}$ progression and therapeutic targets.

Circular ribonucleic acids (circRNAs) are a class of stable, conserved ribonucleic acid (RNA) molecules [8, 9]. Recent studies have indicated that circRNAs encode small peptides [10, 11]. Most studies have proven that circRNAs act as sponges of microRNAs (miRNAs) to further regulate the development of multiple human diseases [8, 12-16]. In human BC, many circRNAs have been discovered and proven to be related to the development of BC [17]. Notably, a meta-analysis indicated that circRNAs have diagnostic value and may be potential diagnostic biomarkers for $\mathrm{BC}$ [18]. However, research on the mechanisms of circRNAs in $\mathrm{BC}$ progression remains limited. In our previous study, a novel circRNA, hsa_circ_0043278, was identified and found to be downregulated in BC tissues [19]. In addition, we predicted five putative target miRNAs. However, the underlying mechanisms of hsa_circ_0043278 remain to be elucidated. Thus, these findings prompted our interest in revealing the association between hsa_circ_0043278 and $\mathrm{BC}$ development.

In the present study, the low expression of hsa circ_0043278 in BC cell lines was examined. Moreover, its target miRNA, miR-455-3p, has been considered an oncogene in $\mathrm{BC}$ owing to its inhibitory effect on etoposide-induced gene 24 (EI24) expression [20]. EI24 has also been reported to have a role in suppressing tumour progression by inhibiting NF- $\mathrm{BB}$ activity [21]. Hence, based on the aforementioned information, we hypothesized that hsa_circ_0043278 might influence BC development by targeting miR-455-3p and regulating EI24. Collectively, our results showed that hsa_circ_0043278 might act as a tumour suppressor gene in $\mathrm{BC}$ progression. Exploration of its molecular mechanism could be valuable for $\mathrm{BC}$ diagnosis and therapy.

\section{Materials and methods Tissue specimens}

A total of 50 pairs of $\mathrm{BC}$ tissues and matched normal tissues were collected from $\mathrm{BC}$ patients at The First Affiliated Hospital of China Medical University (from January 2005 to December 2012). All patients were diagnosed with primary $\mathrm{BC}$, female, between 27 and 81 years old, and did not receive chemotherapy or radiotherapy before surgery. Tissue specimens were obtained after surgical resection and stored at $-80{ }^{\circ} \mathrm{C}$ in an ultralow-temperature freezer (Haier, China). The matched normal tissues were collected from regions more than $5 \mathrm{~cm}$ outside the edge of $\mathrm{BC}$ tissues. The study was approved by the Ethics Committee of The First Affiliated Hospital of China Medical University. All procedures performed in this study involving specimen collection and experiments were in accordance with the Declaration of Helsinki. Written informed consent was obtained from all patients.

\section{Cell culture}

Human BC cell lines (MDA-MB-231 and MCF-7) and a normal mammary epithelial cell line (MCF-10A) were purchased from the Cell Bank of the Chinese Academy of Sciences. The MDA-MB-468, BT-549, SK-BR-3, T47D and HEK 293T cell lines were maintained in our laboratory. MDA-MB-231 cells were cultured in Dulbecco's modified Eagle's medium (DMEM; Gibco, Carlsbad, CA, USA) containing $10 \%$ foetal bovine serum (FBS; HyClone, Logan, UT, USA). MCF-7 cells were cultured in minimum essential medium (MEM; Gibco) containing 10\% FBS and $0.01 \mathrm{mg} / \mathrm{ml}$ insulin from bovine pancreas (Aladdin, Shanghai, China). Michigan Cancer Foundation-10A (MCF-10A) cells were cultured in mammary epithelial basal medium (MEBM; iCell Bioscience, Shanghai, China). The other cell lines were cultured appropriately. All these cell lines were maintained at $37^{\circ} \mathrm{C}$ in a humidified incubator with $5 \%$ carbon dioxide $\left(\mathrm{CO}_{2}\right)$.

\section{qRT-PCR}

Total RNA from tissues or cells was extracted with a simple Total RNA Kit (BioTeke, Beijing, China) following the manufacturer's instructions. The purity and concentration of the RNA were determined using a NanoDrop 2000 spectrophotometer (Thermo Scientific, Waltham, MA, USA). Complementary deoxyribonucleic acid (cDNA) was reverse transcribed from the total RNA using Moloney Murine Leukaemia Virus (M-MLV) Reverse Transcriptase (BioTeke). Quantitative reverse transcription-polymerase chain reaction (qRT-PCR) was performed in an Exicycler ${ }^{\mathrm{TM}} 96$ RT-PCR instrument (BIONEER, Daejeon, Korea) using the SYBR Green method (Takara Bio, Dalian, China). $\beta$-Actin was used as the control for the normalization of circRNA and messenger RNA (mRNA) levels, while U6 was used for normalization of miRNA levels. Table S1 shows the primers for gene amplification, which were designed with Primer Premier 5 (PREMIER Biosoft, USA) and synthesized by Sangon Biotech (Shanghai, China). The relative expression of genes was analysed using the $2^{-\triangle \mathrm{CT}}$ method. All the experiments were repeated three times.

\section{Vector construction and cell transfection}

To overexpress hsa_circ_0043278, the mature sequence of hsa_circ_0043278 (chr17, 35797838-35800763) was synthesized and then cloned into the pCD5-ciR vector (GenScript, Nanjing, China) and was named "ovcirc". A mock vector without the hsa_circ_0043278 sequence (ov-NC) served as the negative control. To knock down hsa_circ_0043278, two small interfering 
RNAs (siRNAs) targeting the back-splice junction site of hsa_circ_0043278 and a negative control siRNA (si-NC) were synthesized (GenScript). Consequently, qRT-PCR proved that siRNA-2 was the most effective siRNA, and it was used to construct the siRNA plasmid (Fig. S1). The shRNA against hsa_circ_0043278 and the negative control shRNA were synthesized and cloned into the pRNAH1.1 vector, and the resulting vectors were named "sh-circ" and "sh-NC", respectively. The sequences of the vectors and siRNAs were designed with the online NCBI database (https://www.ncbi.nlm.nih.gov/). The miR-455-3p mimics and inhibitors were purchased from GenePharma (Shanghai, China). Vectors, mimics, and inhibitors were transiently transfected into $\mathrm{BC}$ cells with Lipofectamine 2000 (Invitrogen, Carlsbad, CA, USA) following the manufacturer's protocols. The sequences of the siRNAs and shRNAs are shown in Table S2. The miR-455-3p inhibitor and negative control sequences are listed in Table S3.

\section{Western blotting}

Proteins from tissues and cells were extracted using radioimmunoprecipitation assay (RIPA) lysis buffer (Beyotime, Haimen, China) supplemented with $1 \mathrm{mM}$ phenylmethylsulfonyl fluoride (PMSF; Beyotime). A total of $40 \mu \mathrm{g}$ (in $20 \mu \mathrm{l}$ ) of protein from each sample was separated by sodium dodecyl sulfate-polyacrylamide gel electrophoresis (SDS-PAGE) and was then transferred onto polyvinylidene difluoride (PVDF) membranes (Millipore, Bedford, MA, USA). Next, the membranes were incubated with $5 \%$ milk at room temperature for $1 \mathrm{~h}$. Then, they were incubated with the appropriate primary antibody overnight at $4{ }^{\circ} \mathrm{C}$ and then with a horseradish peroxidase-conjugated secondary antibody $(1: 5,000$; Wanleibio, Shenyang, China). The specific primary antibodies included anti-EI24 (1:1000, Proteintech, Wuhan, China) and anti-NF-kB (P65) (1:500, Wanleibio) antibodies. $\beta$-Actin and histone H3 (1:1000, Wanleibio) served as the internal controls. Immune complexes were finally visualized with an enhanced chemiluminescence system (Beyotime).

\section{3-(4,5-Dimethylthiazol-2-yl)-2,5-diphenyl-tetrazolium bromide (MTT) assay}

Cells were seeded in 96-well plates at a density of $3 \times 10^{3}$ cells per well. MTT solution $(5 \mathrm{mg} / \mathrm{mL}$; Sigma-Aldrich, St. Louis, Missouri) was added to each well at the indicated time points $(0,24,48$, and $72 \mathrm{~h})$, and the plates were incubated at $37{ }^{\circ} \mathrm{C}$ in $5 \% \mathrm{CO}_{2}$ for $4 \mathrm{~h}$. Next, the medium was removed, and $200 \mu \mathrm{L}$ of dimethyl sulfoxide (DMSO) was added to dissolve the formazan crystals.
The optical density at $570 \mathrm{~nm}$ was recorded using an automatic microplate reader (BioTek, Vermont, USA).

\section{Colony formation assay}

Briefly, cells in each group were seeded in $35-\mathrm{mm}$ dishes at 500 cells/dish and incubated at $37{ }^{\circ} \mathrm{C}$ in $5 \% \mathrm{CO}_{2}$ for 2 weeks. The cells were fixed and stained with Giemsa solution (KeyGEN BioTECH, Jiangsu, China). Colonies consisting of at least 50 cells were counted and photographed under a microscope.

\section{Wound healing assay}

Cells were seeded $\left(6 \times 10^{5}\right.$ cells/well $)$ and grown in 6-well plates for $48 \mathrm{~h}$ post transfection. Next, the cells were cultured in serum-free culture medium and treated with 1 $\mu \mathrm{g} / \mathrm{ml}$ mitomycin C (Sigma-Aldrich) for $1 \mathrm{~h}$. Wounds were created in the middle of the wells using $200-\mu \mathrm{L}$ pipette tips. The cells were then washed and cultured in serum-free culture medium, and wound closure was monitored by imaging with a phase contrast microscope (Motic, Xiamen, China). After culture at $37^{\circ} \mathrm{C}$ in $5 \% \mathrm{CO}_{2}$ for $48 \mathrm{~h}$, the widths of the wounds were measured again, and the migration rates were calculated in each group.

\section{Migration and invasion assays}

For the migration assays, $2 \times 10^{4}$ cells were seeded in the upper chambers of a Transwell plate (Corning Incorporated, Corning, NY, USA) in $200 \mu \mathrm{L}$ of serum-free medium. An $800-\mu \mathrm{L}$ volume of medium containing $20 \%$ FBS was added to the lower chambers. The pore size of the Transwell membrane was $8.0 \mu \mathrm{m}$. After incubation at $37{ }^{\circ} \mathrm{C}$ in $5 \% \mathrm{CO}_{2}$ for $24 \mathrm{~h}$, the cells that migrated to the bottom of the filter were fixed with $4 \%$ paraformaldehyde and stained with $0.5 \%$ crystal violet (Amresco, USA) and were then photographed and counted under an inverted phase contrast microscope (Motic).

For the invasion assays, $2 \times 10^{4}$ cells in $200 \mu \mathrm{L}$ of serumfree medium were seeded in the upper chambers of a Transwell plate with a Matrigel-coated membrane (BD Biosciences, NJ, USA). An $800-\mu \mathrm{L}$ volume of medium containing $20 \%$ FBS was added to the lower chambers. The pore size of the Transwell membrane was $8.0 \mu \mathrm{m}$. After incubation at $37{ }^{\circ} \mathrm{C}$ in $5 \% \mathrm{CO}_{2}$ for $24 \mathrm{~h}$, the cells in the lower compartment were fixed with $4 \%$ paraformaldehyde and stained with $0.5 \%$ crystal violet (Amresco) and were then photographed and counted under an inverted phase contrast microscope (Motic).

\section{Xenograft experiment}

A total of 18 two-month-old female BALB/c (nu/nu) mice were purchased from Wanleibio (Shenyang, China). The animal care and experimental procedures were approved by the Experimental Animal Ethics Committee 
of The First Affiliated Hospital of China Medical University. All animal procedures were performed in accordance with the Guide for the Care and Use of Laboratory Animals published by the National Institutes of Health (NIH, Bethesda, Maryland, USA). We made great attempts to reduce the pain experienced by the animals. Mice were adaptively fed for 1 week under specific pathogen-free conditions. To establish xenograft tumours, 0.2 $\mathrm{mL}\left(5 \times 10^{7}\right.$ cells $\left./ \mathrm{ml}\right)$ of MDA-MB-231 cells stably transfected with the hsa_circ_0043278 overexpression vector or MCF-7 cells stably transfected with hsa_circ_0043278 shRNA was subcutaneously injected into the right axillae of the mice, and mice injected with mock vector or shRNA served as negative controls $(n=3$ mice in each group; 18 mice total). The tumour sizes were measured once a week with a calliper. The tumour volumes were calculated using the following formula: 1/2 (length $\times$ width $^{2}$ ). In brief, mice were sacrificed in the 4th week, and the tumours were then excised, photographed and weighed.

\section{Immunohistochemistry (IHC)}

The expression of NF-kB (P65) in the paraffin-embedded xenograft tissue was detected by immunohistochemistry. Tissues were incubated with the anti-NF-kB (P65) (1:200; Wanleibio) primary antibody at $4{ }^{\circ} \mathrm{C}$ overnight and then with the secondary antibody at $37^{\circ} \mathrm{C}$ for $60 \mathrm{~min}$ and an HRP-labelled streptavidin solution for $10 \mathrm{~min}$. Next, tissues were stained by diaminobenzidine (DAB) (Solarbio, China) and observed under a microscope. The mean density of P65 in the tissues was calculated using Image-Pro software.

\section{Dual-luciferase reporter assay}

The sequences of wild-type hsa_circ_0043278 or a mutant without miR-455-3p binding sites were synthesized and subcloned into the luciferase reporter vector pmirGLO (GenScript); the resulting vectors were named "circ-WT" and "circ-Mut", respectively. HEK 293T cells were cotransfected with the vectors and miR-455-3p mimics or negative controls. Relative luciferase activity was measured with a Dual-Luciferase Assay Kit (KeyGEN BioTECH) in accordance with the manufacturer's protocols after $48 \mathrm{~h}$ of incubation. Luciferase activity was measured using a Tecan Infinite M200 Pro luminometer (Tecan, Männedorf, Switzerland).

\section{Statistical analysis}

Statistical analysis was performed using GraphPad Prism 7.0 software (GraphPad Software, La Jolla, CA, USA). The data for two groups were compared using a $t$ test with the Wilcoxon signed-rank test. Comparisons of the means among multiple groups were performed by one-way analysis of variance (ANOVA) with the Kruskal-Wallis test. Pearson correlation coefficients were calculated for correlation analyses. Continuous data are presented as the mean \pm standard deviation values (SD). A $P$ value less than 0.05 was considered to indicate statistical significance. All experiments were repeated three times.

\section{Results \\ hsa_circ_0043278 was downregulated in BC tissues and cell lines}

In our previous study, hsa_circ_0043278 was found to be downregulated 43 -fold in $\mathrm{BC}$ tissues compared to matched normal tissues by microarray analysis. In addition, hsa_circ_0043278 was found to be downregulated in BC tissues compared to matched normal tissues by qRTPCR [19]. In this study, BC cell lines (MDA-MB-231, MDA-MB-468, BT-549, MCF-7, SK-BR-3, and T47D) and MCF-10A normal breast epithelial cells were used to verify the differential expression of hsa_circ_0043278. The cDNA of hsa_circ_0043278 was amplified from HEK 293T cells and confirmed by DNA sequencing (Fig. 1A). As shown in Fig. 1B, the expression of hsa circ_0043278 in BC cell lines was lower than that in the normal breast epithelial cell line MCF-10A. Moreover, hsa_circ_0043278 expression in TNBC cell lines (MDAMB-231, MDA-MB-468, and BT-549) was much lower than that in cell lines of other subtypes of BC (MCF-7, SK-BR-3, and T47D). Thus, we chose MDA-MB-231 and MCF-7 cells, which had the lowest and highest expression of hsa_circ_0043278 among the BC cell lines, respectively, for our next study.

\section{hsa_circ_0043278 inhibits BC cell viability, migration and invasion}

To examine the biological role of hsa_circ_0043278 in BC cells, an hsa_circ_0043278 overexpression vector and a shRNA vector targeting hsa_circ_0043278 were constructed (Fig. 2A). Next, we upregulated hsa_circ_0043278 in MDA-MB-231 cells and downregulated it in MCF-7 cells. The expression of hsa circ_0043278 was examined by qRT-PCR (Fig. 2B). Since hsa_circ_0043278 is spliced from the transcriptional adapter 2-alpha (TADA2A) gene (chr17, 35766977-35839830), the expression of the linear transcript TADA2A was also verified by qRT-PCR. The results demonstrated that there was no effect on the expression of TADA2A when hsa_circ_0043278 was overexpressed or knocked down in BC cells (Fig. 2C). Moreover, MTT assays were performed to examine the influence of hsa_circ_0043278 on the viability of BC cells. The results showed that upregulation of hsa circ_0043278 markedly decreased the viability of MDAMB-231 cells at $72 \mathrm{~h}(P<0.01)$, whereas downregulation 

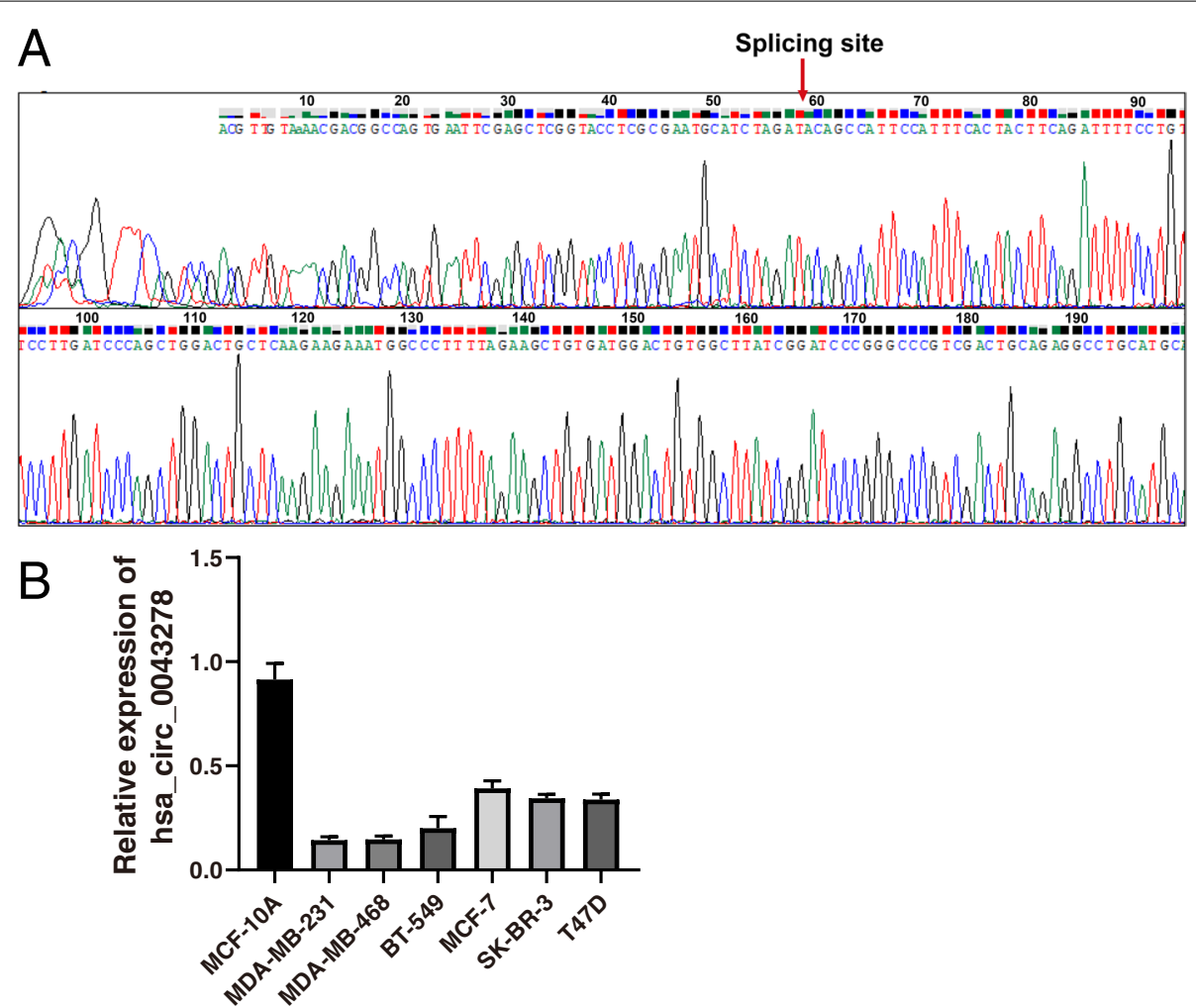

Fig. 1 Hsa_circ_0043278 was downregulated in BC tissues and cell lines. A The PCR products of hsa_circ_0043278 were verified by DNA sequencing. The red arrow shows the back-splice junction site of hsa_circ_0043278. B The levels of hsa_circ_0043278 in MCF-10A cells and BC cell lines were determined by qRT-PCR. The data are presented as the mean \pm standard deviation values $(n=3)$

of hsa_circ_0043278 significantly increased the viability of MCF-7 cells $(P<0.01$; Fig. 2D). In addition, the colony-forming ability of MDA-MB-231 cells was markedly reduced after upregulation of hsa_circ_0043278 $(P<0.05)$ and was significantly increased in MCF-7 cells by downregulation of hsa_circ_0043278 $(P<0.05$; Fig. 2E). To explore the effects of hsa_circ_0043278 on cell migration and invasion, wound healing assays and Transwell assays were performed on BC cells. As shown in Fig. 2F, the wound healing assays revealed that the migration rate of MDA-MB-231 cells was markedly reduced after upregulation of hsa_circ_0043278 $(P<0.001)$. Conversely, the migration rate of MCF-7 cells was markedly increased by downregulation of hsa_circ_0043278 $(P<0.01)$. Similarly, the results of the Transwell assays showed that upregulation of hsa_circ_0043278 significantly inhibited the migration (Fig. 2G) and invasion (Fig. 2H) of MDA-MB-231 cells, while downregulation of hsa_circ_0043278 in MCF-7 cells resulted in the opposite effects (Fig. 2G-H). These results suggested that hsa_circ_0043278 decreased the viability and inhibited the migration and invasion of $\mathrm{BC}$ cells in vitro.
Hsa_circ_0043278 inhibits the tumorigenesis of BC cells in vivo

To explore the role of hsa_circ_0043278 in tumorigenesis in vivo, we subcutaneously injected MDA-MB-231 cells transfected with the hsa_circ_0043278 overexpression vector (ov-circ) or mock vector (ov-NC) or MCF-7 cells transfected with hsa_circ_0043278 shRNA (sh-circ) or the negative control shRNA (sh-NC) into female nude mice. After 4 weeks, the tumours formed from ov-circ MDA-MB-231 cells were significantly smaller and lighter than the tumours formed in the control group $(P<0.05)$, and the tumours formed from MCF-7 cells transfected with sh-circ were larger and heavier than the tumours formed in the sh-NC group $(P<0.01$; Fig. $3 \mathrm{~A}-\mathrm{B})$. Moreover, the tumour volume in the ov-circ group increased at a significantly lower rate than that in the ov-NC group every week, and the tumours in the ov-circ group were significantly smaller than those in the ov-NC group at the 4th week $(P<0.05)$. Conversely, downregulation of hsa_circ_0043278 significantly promoted tumour growth $(P<0.01$; Fig. 3C). Furthermore, NF-kB (P65) is considered to play an important role in promoting tumour progression and invasion [22]. In the canonical pathway, 


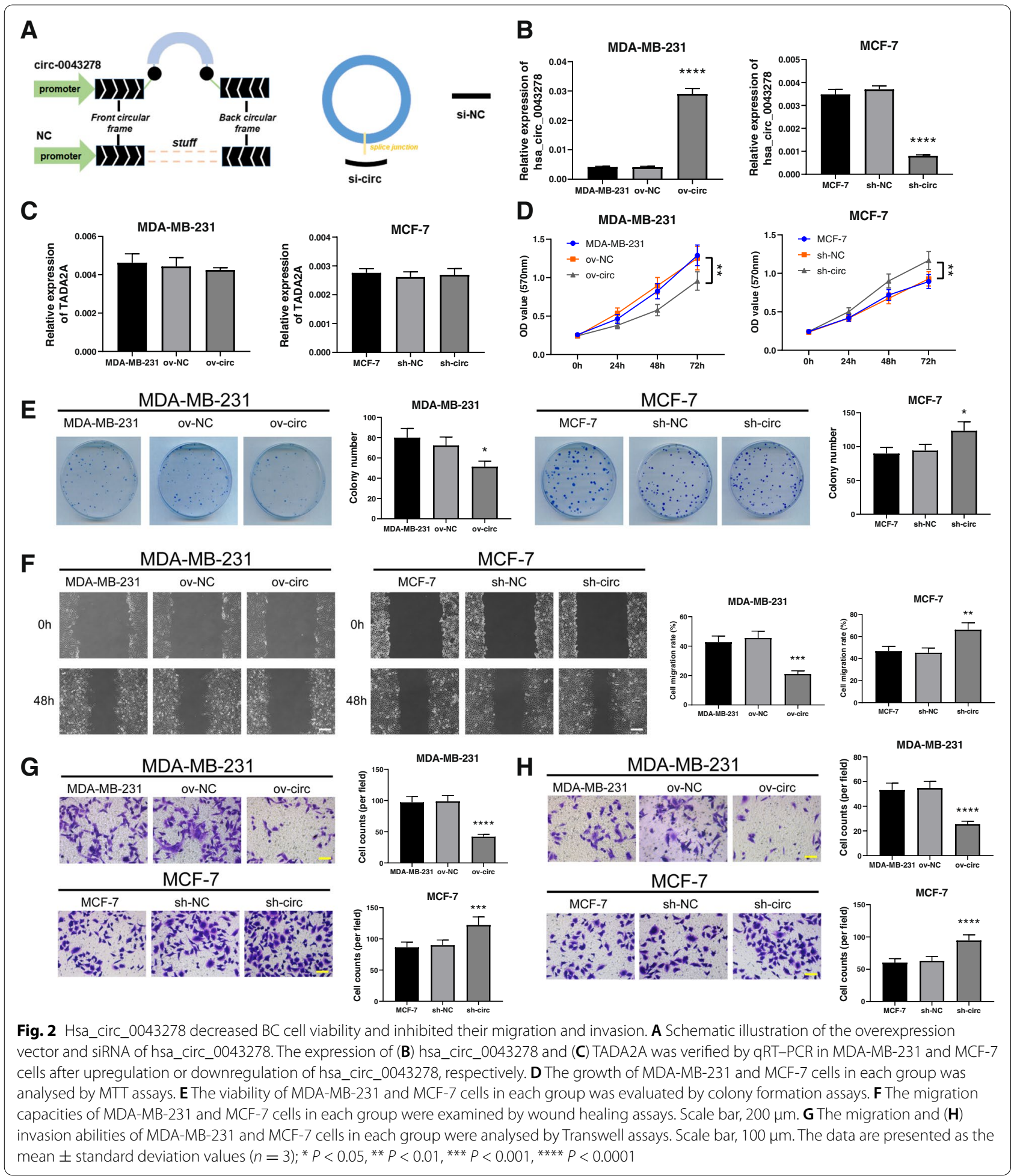

$\mathrm{NF}-\mathrm{kB}$ is translocated to the nucleus and activated after IкB $\alpha$-mediated phosphorylation in an IKK $\beta$ - and NEMO-dependent manner [23]. In addition, NF- $\mathrm{BB}$ has been reported to be inhibited by EI24 in BC cells [21].
Thus, inhibition of nuclear NF-kB might be effective for the inhibition of $\mathrm{BC}$ progression. To determine the influence of hsa_circ_0043278 on P65, immunohistochemical staining of tumour tissues was performed. The results 


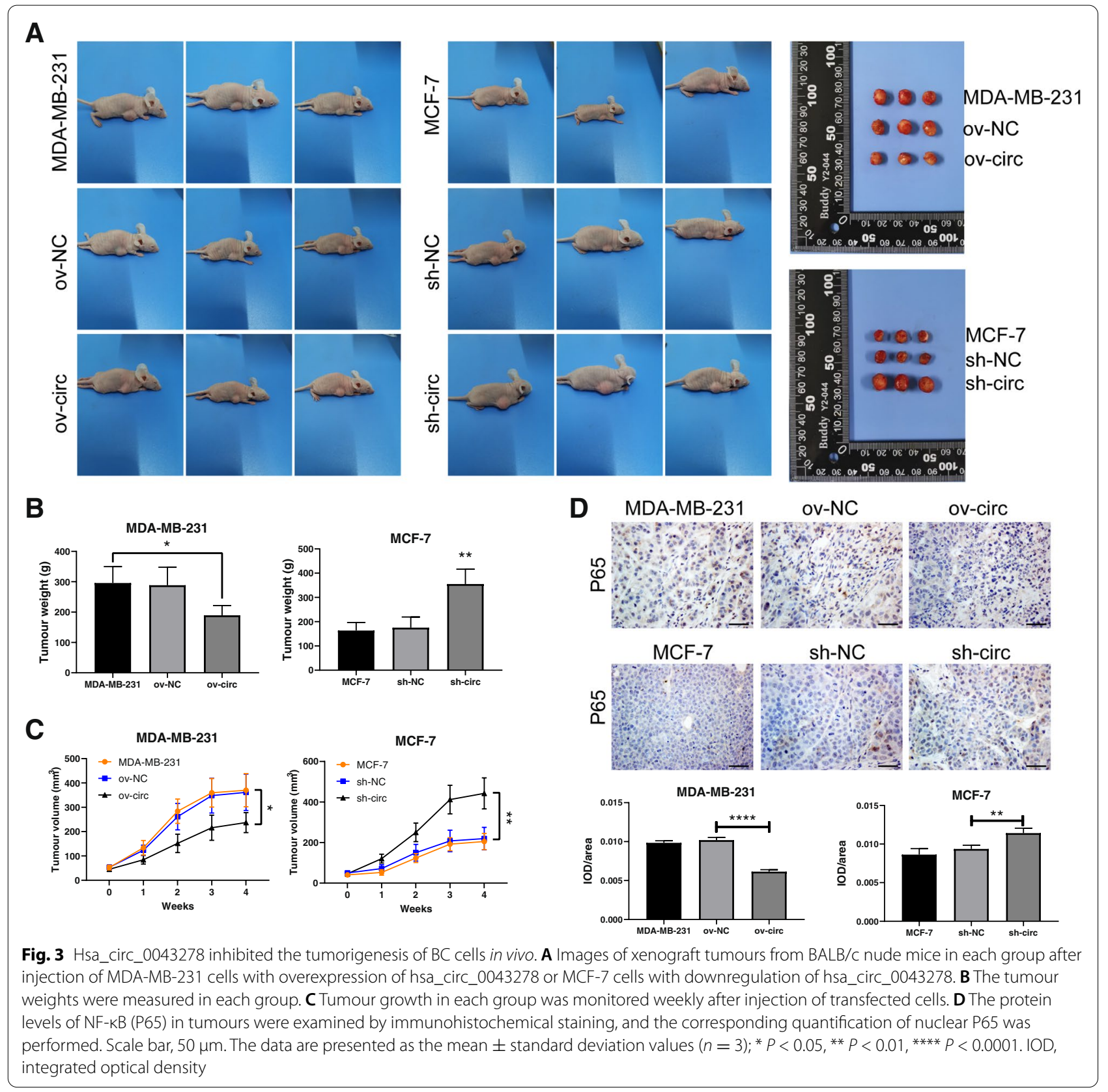

showed that upregulation of hsa_circ_0043278 notably reduced the expression of $\mathrm{P} 65$ in the nucleus $(P<0.0001)$; in contrast, downregulation of hsa_circ_0043278 significantly increased the level of P65 in xenograft tumour tissues $(P<0.01$; Fig. 3D). These results indicated that hsa circ_0043278 might inhibit tumour growth and affect NF-kB activation in vivo.

\section{Hsa_circ_0043278 functions as a sponge for miR-455-3p}

In our previous study, we predicted the 5 miRNAs most likely to interact with hsa_circ_0043278: miR-455-3p,
miR-103a-2-5p, miR-302b-3p, miR-302c-3p, and miR520d-3p [19]. To explore the molecular mechanism of hsa_circ_0043278 in BC, qRT-PCR was performed to examine the expression levels of miRNAs after upregulation or downregulation of hsa_circ_0043278 in BC cells. Among the 5 abovementioned miRNAs, miR-455-3p exhibited markedly decreased expression compared to that in the control group when hsa_circ_0043278 was overexpressed in MDA-MB-231 cells $(P<0.0001)$, whereas its expression was significantly elevated after knockdown of hsa_circ_0043278 in MCF-7 cells $(P<0.0001$; 
Fig. 4A). The levels of the other 4 predicted miRNAs showed no obvious changes after upregulation of hsa circ_0043278 in MDA-MB-231 cells (Fig. S2). Thus, miR-455-3p might be a target of hsa_circ_0043278 in BC cells. Furthermore, miR-455-3p was determined to be markedly upregulated in $50 \mathrm{BC}$ tissues compared with the matched normal tissues by qRT-PCR $(P<0.001$; Fig. 4B). Additionally, Pearson correlation analysis of these $50 \mathrm{BC}$ tissue samples showed a significant negative correlation between the levels of miR455-3p and hsa_circ_0043278 $(r=-0.3468, P=0.0136$; Fig. 4C). Similarly, the level of miR-455-3p was elevated in all $\mathrm{BC}$ cell lines compared to MCF-10A cells, as determined by qRT-PCR (Fig. 4D). Thus, to determine whether hsa_circ_0043278 serves as a competing endogenous RNA (ceRNA) for miR-455-3p, a dual-luciferase reporter assay was performed in HEK 293T cells. Bioinformatics prediction analysis showed that there were two potential binding sites between miR-455-3p and hsa_circ_0043278 [19]. We subcloned the full-length wild-type hsa_circ_0043278 sequence (circ-WT) and two mutant versions without miR-455-3p binding sites (circ-Mut) into luciferase reporter vectors (Fig. 4E-F). We found that in the circWT groups, transfection with the miR-455-3p mimics markedly reduced the luciferase activity compared to that in the miR-455-3p NC group $(P<0.01)$. Moreover, the miR-455-3p mimics did not decrease the luciferase activity in either circ-Mut group (Fig. 4E-F). This suggested that hsa_circ_0043278 might function as a sponge for miR-455-3p through the two predicted binding sites.

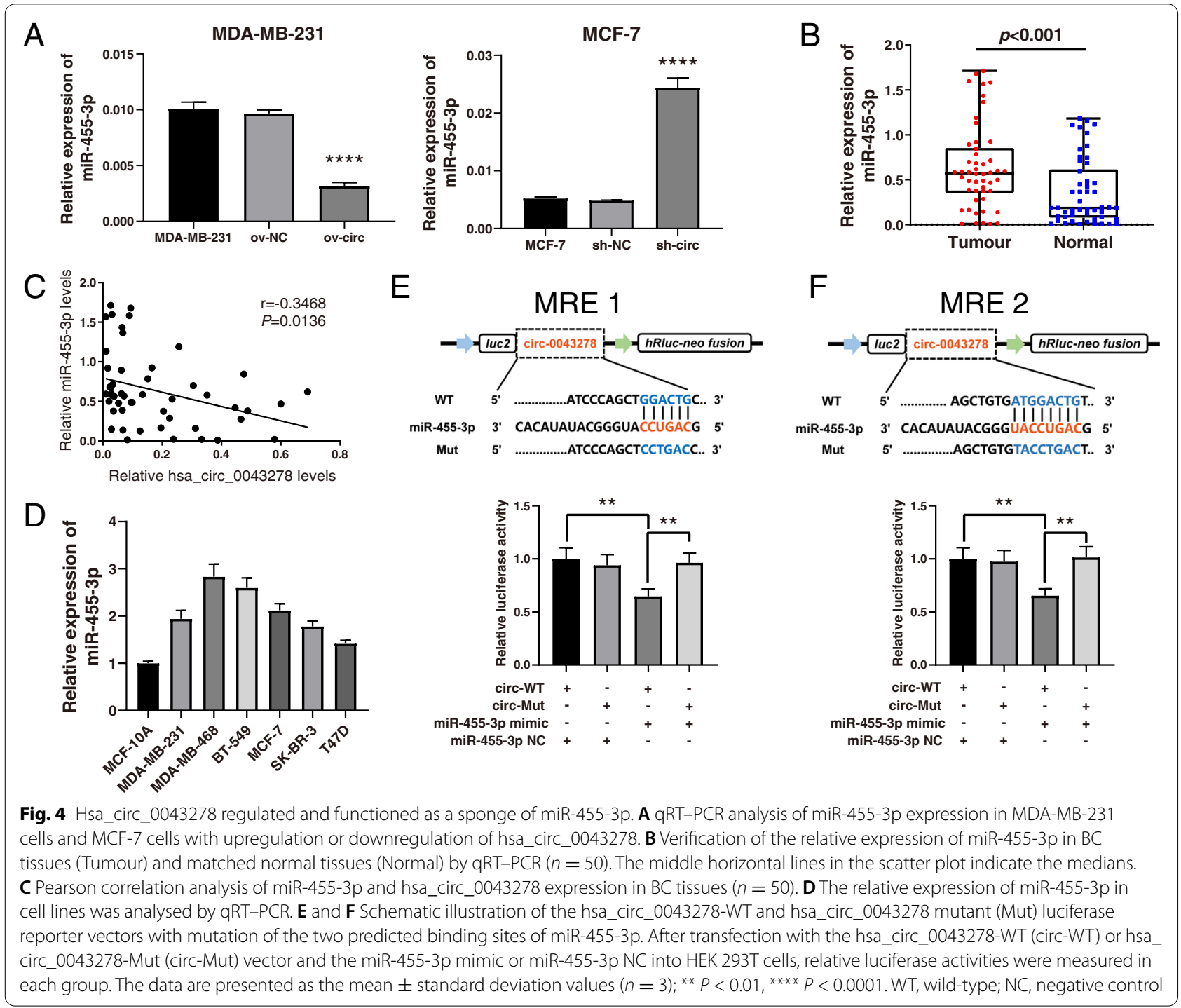




\section{El24 is a target of miR-455-3p and is indirectly regulated by hsa_circ_0043278}

To explore the mechanism of hsa_circ_0043278 in BC, we predicted the downstream targets of miR-455-3p. In our previous study, two potential binding sites between miR-455-3p and hsa_circ_0043278 were predicted [19]. According to the TargetScan and miRanda prediction results in this study, EI24 and hsa_circ_0043278 were most likely to share the same miRNA response element (MRE) for miR-455-3p (Fig. 5A). In addition, EI24 was reported to be a direct target of miR-455-3p and to act as a tumour suppressor gene in triple-negative $\mathrm{BC}$ (TNBC) [20]. Thus, we hypothesized that EI24 might be indirectly regulated by hsa_circ_0043278 through miR-455-3p. Furthermore, EI24 expression was verified to be decreased in the $50 \mathrm{BC}$ tissues compared to the matched normal tissues by qRT-PCR $(P<0.01$; Fig. $5 \mathrm{~B})$. Moreover, the expression level of EI24 was positively associated with the hsa_circ_0043278 expression level in Pearson correlation analysis $(r=0.5359, P<0.0001$; Fig. 5C). The expression of EI24 was also decreased in BC cell lines compared to MCF-10A cells (Fig. 5D). After verifying that hsa_circ_0043278 can directly bind to miR455-3p, we then examined whether hsa_circ_0043278 affects EI24 expression to understand the role of the hsa_circ_0043278/miR-455-3p/EI24 axis in BC cells. The qRT-PCR results indicated that overexpression of hsa circ_0043278 significantly increased the level of EI24 in MDA-MB-231 cells $(P<0.0001)$ and that knockdown of hsa_circ_0043278 markedly decreased the level of EI24 in MCF-7 cells $(P<0.0001$; Fig. 5 E). Similarly, western blot analysis indicated that the protein level of EI24 was also positively regulated by hsa_circ_0043278 in the 2 BC cell lines $(P<0.0001$; Fig. $5 \mathrm{~F})$. These results showed that hsa circ_0043278 might regulate the expression level of EI24 by functioning as a sponge for miR-455-3p in BC.

Next, we examined whether hsa_circ_0043278 affects the expression of P65 in the nucleus of BC cells. Western blot analysis suggested that upregulation of hsa circ_0043278 markedly decreased the protein level of $\mathrm{P} 65$ in the nucleus $(P<0.0001)$ and increased it in the cytosol in MDA-MB-231 cells $(P<0.0001)$, while knockdown of hsa_circ_0043278 resulted in the opposite effects in MCF-7 cells (Fig. 5G). These results revealed that hsa_circ_0043278 influenced the expression of the downstream gene of EI24 and might act as a tumour suppressor by inhibiting NF- $\mathrm{kB}$ in BC cells.

\section{Validation of hsa_circ_0043278-mediated inhibition of BC progression through the hsa_circ_0043278/miR-455-3p/ El24 axis}

After verifying that hsa_circ_0043278 directly binds to miR-455-3p and regulates the EI24 mRNA and protein levels, it was essential to explore whether hsa_circ_0043278 acts in BC progression through the hsa_circ_0043278/miR-455-3p/EI24 axis. miR-455-3p mimics and inhibitors were designed and cotransfected into MDA-MB-231 and MCF-7 cells. After that, the level of miR-455-3p was examined by qRT-PCR (Fig. 6A). The MTT assay showed that upregulation of hsa_circ_0043278 significantly decreased the viability of MDA-MB-231 cells and that this effect could be suppressed by cotransfection of the miR-455-3p mimic. Furthermore, downregulation of hsa_circ_0043278 significantly increased the viability of MCF-7 cells, but this effect was abolished by cotransfection of the miR-455-3p inhibitor (Fig. 6B). Additionally, the wound healing assays demonstrated that the miR-455-3p mimic counteracted the reduction in the migration rate of MDA-MB-231 cells induced by overexpression of hsa_circ_0043278, while the miR-455-3p inhibitor reversed the increase in the migration rate of MCF-7 cells induced by silencing hsa circ_0043278 (Fig. 6C). Similarly, the Transwell assays revealed that the influences of upregulating or silencing hsa_circ_0043278 on BC cell migration and invasion were rescued by the miR-455-3p mimic and inhibitor, respectively (Fig. 6D-E).

Subsequently, the qRT-PCR and western blot results revealed that upregulation of hsa_circ_0043278 increased the mRNA and protein levels of EI24 in MDAMB-231 cells, while downregulation of hsa_circ_0043278 decreased the mRNA and protein levels of EI24 in MCF-7 cells. These effects were reversed by cotransfection of the miR-455-3p mimic or inhibitor (Fig. 7A-B).

\footnotetext{
(See figure on next page.)

Fig. 5 El24 was directly targeted by miR-455-3p and regulated by hsa_circ_0043278. A Prediction of miR-455-3p binding sites in El24 and hsa_ circ_0043278 using TargetScan and MiRanda. The binding sites are presented as solid lines. B Verification of the relative expression of El24 in BC tissues (Tumour) and matched normal tissues (Normal) by qRT-PCR $(n=50)$. The middle horizontal lines in the scatter plot indicate the medians. C Pearson correlation analysis of El24 and hsa_circ_0043278 expression in BC tissues ( $n=50)$. D Relative mRNA levels of El24 in cell lines were determined by qRT-PCR. E qRT-PCR and (F) western blot analysis of the El24 mRNA level in MDA-MB-231 cells and MCF-7 cells after upregulation or downregulation of hsa_circ_0043278. (The images of the western blot bands were cropped from the images shown in Figs. S3 and S4). G The relative protein level of NF-kB (P65) in the cytosol and nucleus of cells in each group were determined by western blot analysis. (The images of the western blot bands were cropped from the images shown in Figs. S5, S6, S7, and S8.) The data are presented as the mean \pm standard deviation values $(n=3) ;{ }^{* * * *} P<0.0001$
} 


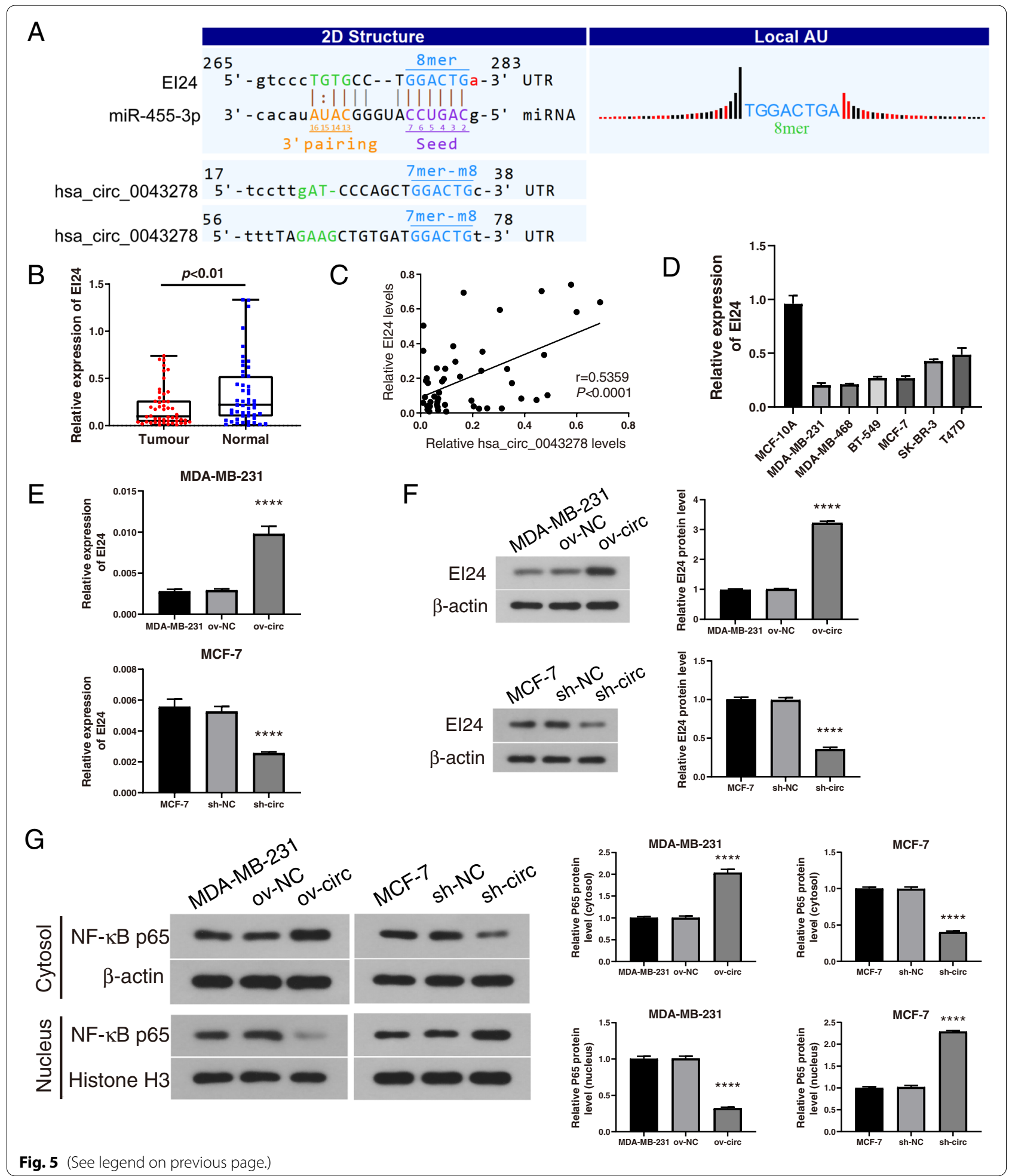

In addition, the level of P65 was significantly decreased in the nucleus after overexpression of hsa_circ_0043278 and increased by downregulation of hsa_circ_0043278; these effects were also counteracted by the miR-455-3p mimic or inhibitor (Fig. 7C). These results showed that hsa_circ_0043278 might function through the hsa circ_0043278/miR-455-3p/EI24 axis to regulate the development of BC. 


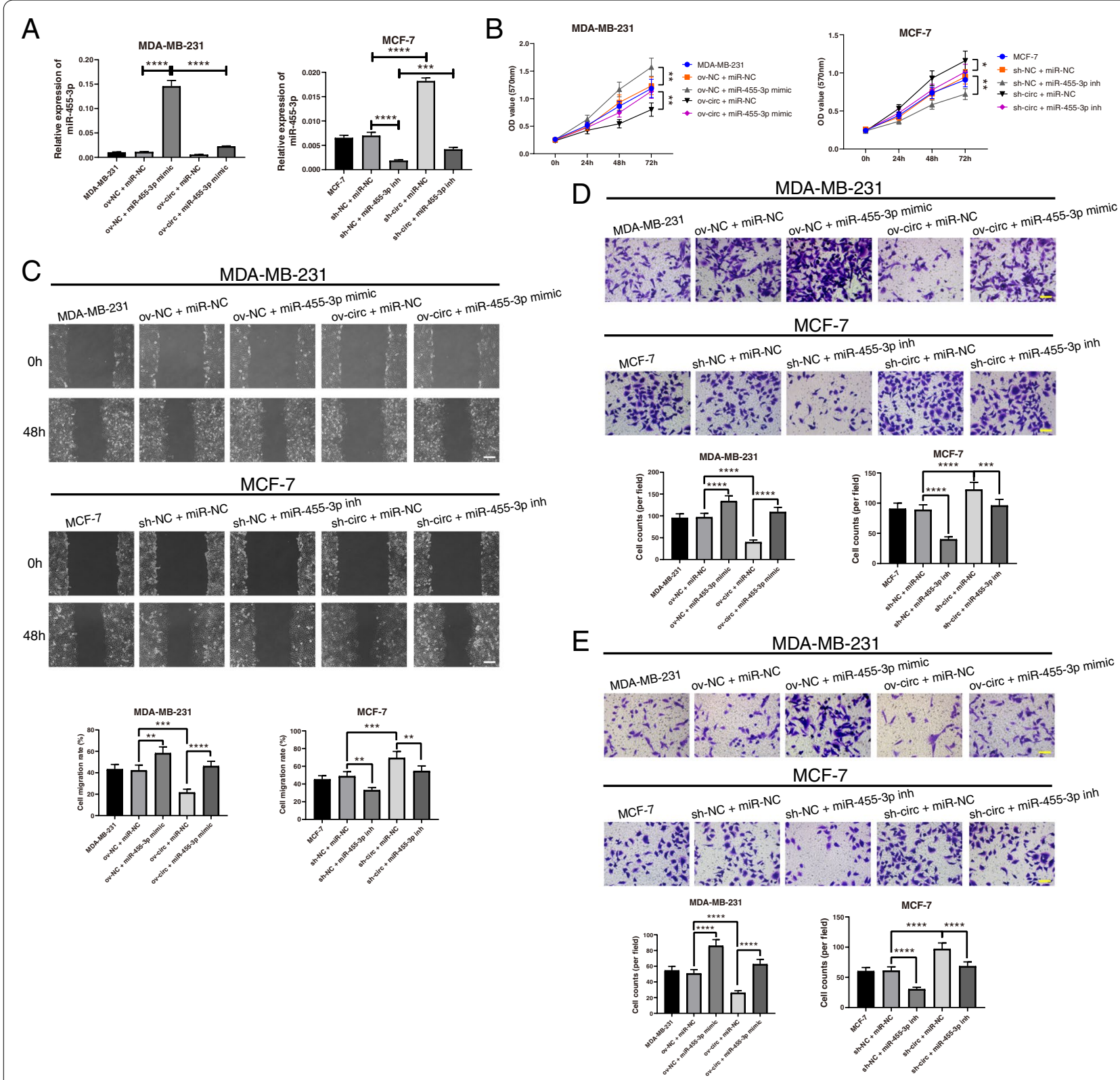

Fig. 6 Hsa_circ_0043278 inhibits BC cell growth, migration and invasion by sponging miR-455-3p. A qRT-PCR was used to examine the relative expression of miR-455-3p in MDA-MB-231 cells after transfection of the hsa_circ_0043278 overexpression vector and the miR-455-3p mimic, as well as in MCF-7 cells after transfection of hsa_circ_0043278 shRNA and the miR-455-3p inhibitor. B The growth of MDA-MB-231 and MCF-7 cells in each group was analysed by MTT assays. C The migration capacities of MDA-MB-231 and MCF-7 cells in each group were examined by wound healing assays. Scale bar, $200 \mu \mathrm{m}$. D and E The migration and invasion abilities of MDA-MB-231 and MCF-7 cells in each group were analysed by Transwell assays. Scale bar, $100 \mu \mathrm{m}$. The data are presented as the mean \pm standard deviation values $(n=3) ;{ }^{*} P<0.05,{ }^{* *} P<0.01$, ${ }^{* *} P<0.001$, ${ }^{* * * *} P<$ 0.0001

\section{Discussion}

$\mathrm{BC}$ represents the most common malignancy and the leading cause of cancer death in women worldwide [24]. Thus, finding a new therapeutic target is an urgent need to improve the survival of BC patients. Sequencing technology has shown that circRNAs are involved in various malignancies and function as diagnostic markers or therapeutic targets $[8,25]$. circRNAs are recognized as a special class of RNA and are increasingly becoming a hotspot of RNA research. However, our knowledge of circRNAs is still limited $[25,26]$. The relationship between circRNAs and $\mathrm{BC}$ has rarely been reported. Discovering 

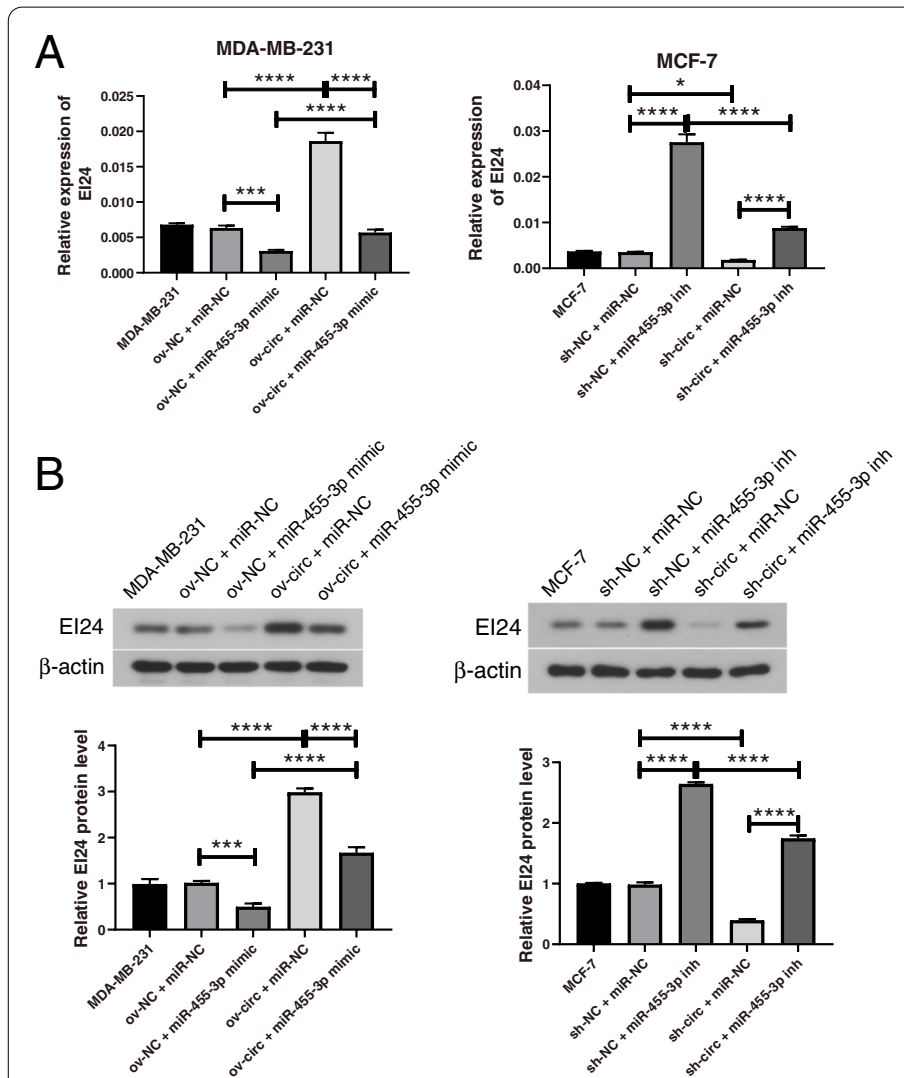
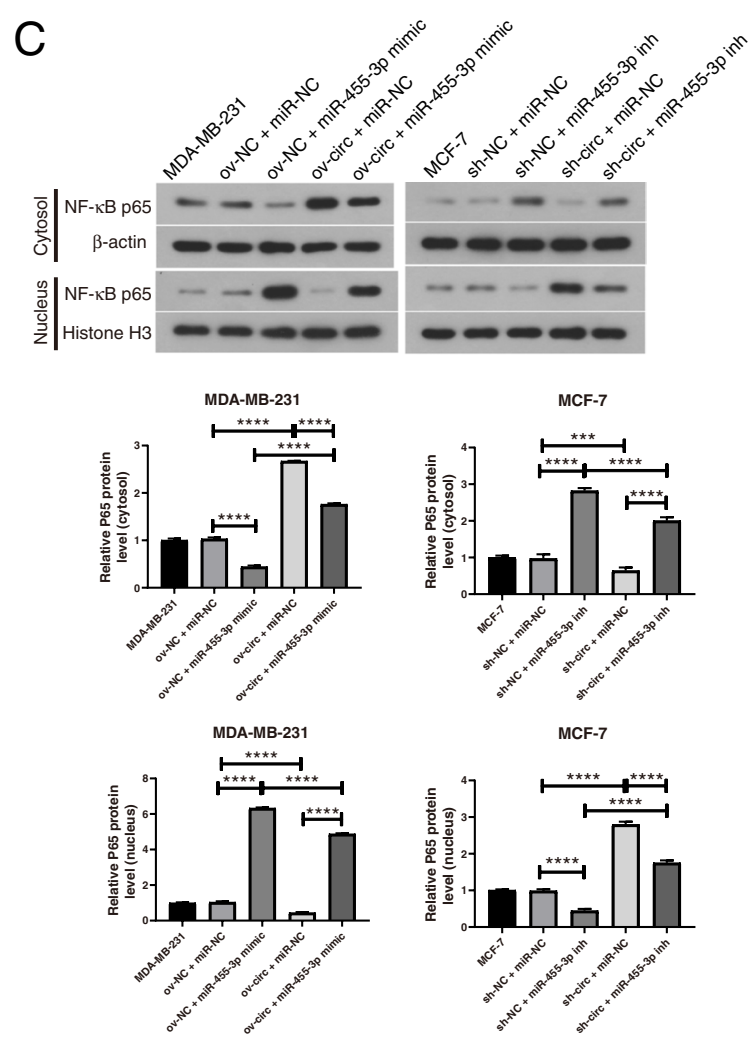

Fig. 7 Hsa_circ_0043278 regulates El24 and NF-kB expression by sponging miR-455-3p. A qRT-PCR and $\mathbf{B}$ western blotting were used to examine the relative expression of EI24 in MDA-MB-231 cells after transfection of the hsa_circ_0043278 overexpression vector and the miR-455-3p mimic as well as in MCF-7 cells after transfection of hsa_circ_0043278 shRNA and the miR-455-3p inhibitor. (The images of the western blot bands were cropped from the images shown in Figs. S9 and S10). C Relative protein levels of NF-KB (P65) in the cytosol or nucleus of MDA-MB-231 and MCF-7 cells in each group were determined by western blot analysis. (The images of the western blot bands were cropped from the images shown in Figs. S11, S12, S13, and S14.) The data are presented as the mean \pm standard deviation values $(n=3) ;{ }^{*} P<0.05,{ }^{* * *} P<0.001,{ }^{* * *} P<0.0001$

the biological roles of circRNAs could be beneficial for the diagnosis and treatment of $\mathrm{BC}$.

Previously, we found that hsa_circ_0043278 was significantly downregulated in $\mathrm{BC}$ tissues. The downregulated expression of hsa_circ_0043278 in BC cell lines relative to normal mammary epithelial cells was verified. These findings led us to discover the correlation between hsa_circ_0043278 and BC. Thus, we selected MCF-7 (with the highest expression of hsa_circ_0043278) and MDA-MB-231 (with the lowest expression of hsa circ_0043278) cells for subsequent research. Functional experiments suggested that overexpression of hsa circ_0043278 significantly decreased the viability and the migration and invasion abilities of BC cells in vitro and decreased tumour growth in vivo, while downregulation of hsa_circ_0043278 showed the opposite effects. These results suggested that hsa_circ_0043278 might act as a tumour suppressor gene in $\mathrm{BC}$ development.

miRNAs have been reported to bind to the 3'UTR and inhibit the expression of their target mRNAs [27-31].
CircRNAs have been generally reported to act as potent miRNA sponges [12] via MREs, thus inhibiting miRNA expression [32, 33]. In our previous study, we predicted that hsa_circ_0043278 contained 2 possible MREs for miR-455-3p [19]. In addition, miR-455-3p has been reported to regulate EI24 expression in TNBC and to serve as an oncogene [20]. Thus, we speculated that hsa_circ_0043278 might function by targeting miR455-3p and regulating EI24 in BC. In our current study, miR-455-3p was found to be overexpressed in $B C$ tissues and cell lines. The expression of miR-455-3p was negatively correlated with that of hsa_circ_0043278 in BC tissues. Moreover, the qRT-PCR and dual-luciferase reporter assay results suggested that hsa_circ_0043278 suppressed the expression of miR-455-3p by directly sponging it. The results proved the relationship between hsa_circ_0043278 and miR-455-3p in BC.

Furthermore, EI24 has been reported to be a target gene of miR-455-3p [20]. It is also considered to act a tumour suppressor gene by inhibiting NF- $\mathrm{kB}$ activity 
[21]. In the current study, EI24 and hsa_circ_0043278 were the most likely circRNA-miRNA target pair to share the same MRE for miR-455-3p. Next, we confirmed that EI24 was also markedly downregulated in $\mathrm{BC}$ tissues and cell lines. Our results showed that overexpression of hsa circ_0043278 increased the EI24 level and suppressed the activity of NF- $\mathrm{kB}$ in $\mathrm{BC}$ cell lines. The rescue experiments demonstrated that inhibiting miR-455-3p partially suppressed the tumour-enhancing effect induced by silencing of hsa_circ_0043278 in MCF-7 cells. The changes in the level of EI24 and the activity of NF- $\mathrm{kB}$ were also partially reversed. Moreover, enhancing miR-455-3p expression showed the opposite effect in MDA-MB-231 cells. These results supported the hypothesis that hsa circ_0043278 acts as a ceRNA of miR-455-3p to decrease the viability and inhibit the migration and invasion of $\mathrm{BC}$ cells. The hsa_circ_0043278/miR-455-3p/EI24 signalling axis might facilitate the progression of $\mathrm{BC}$.

Additionally, we found that hsa_circ_0043278 expression was much lower in TNBC cell lines than in cell lines of other subtypes of $\mathrm{BC}$. Thus, the relationship between TNBC and hsa_circ_0043278 needs to be verified in the future. The precise relationship between hsa circ_0043278 and NF-kB also needs to be elucidated. Furthermore, the number of patient samples needs to be increased. Multicentre trials are also needed to investigate the roles of hsa_circ_0043278.

\section{Conclusions}

This study identified downregulation of hsa_circ_0043278 in BC cells and tissues. Additionally, hsa_circ_0043278 was found to significantly inhibit $\mathrm{BC}$ progression by sponging miR-455-3p, thus influencing the level of EI24 and the activity of NF- $\mathrm{kB}$. Hsa_circ_0043278 may be regarded as a novel prognostic predictor and a potential therapeutic target for BC. The hsa_circ_0043278/miR455-3p/EI24 axis might be a crucial mechanism of BC cell progression.

\section{Abbreviations}

BC: Breast cancer; circRNA: Circular RNA; miRNA: microRNA; 3'UTR: 3'-untranslated region; ceRNA: Competing endogenous RNA; MRE: miRNA response element; IHC: Immunohistochemistry.

\section{Supplementary Information}

The online version contains supplementary material available at https://doi. org/10.1186/s12885-021-08989-w.

Additional file 1: Table S1. The sequences of primers in this study

Additional file 2: Table $\mathbf{S 2}$. The sequences of siRNAs and shRNAs

Additional file 3: Table S3. miR-455-3p inhibitor sequence
Additional file 4: Figure S1. Two siRNAs (siRNA-1 and siRNA-2) targeting the back-splice junction site of hsa_circ_0043278 were constructed, and the expression of hsa_circ_0043278 in MCF-7 cells was analysed by qRTPCR. The data are presented as the mean \pm standard deviation values ( $n$ $=3) ; * * * P<0.0001$

Additional file 5: Figure S2. The expression of miR-103a-2-5p, miR302b-3p, miR-302c-3p, and miR-520d-3p was measured by qRT-PCR after overexpression of hsa_circ_0043278 in MDA-MB-231 cells. The data are presented as the mean \pm standard $(n=3)$.

Additional file 6: Figure S3. Western blot bands of EI24 (A) and $\beta$-actin (B) after hsa_circ_0043278 overexpression in MDA-MB-231 cells.

Additional file 7: Figure S4. Western blot bands of $\beta$-actin (A) and El24 (B) after hsa_circ_0043278 downregulation in MCF-7 cells.

Additional file 8: Figure S5. Western blot bands of NF-KB (P65) (A) and $\beta$-actin (B) in the cytosol after hsa_circ_0043278 overexpression in MDAMB-231 cells.

Additional file 9: Figure S6. Western blot bands of histone $\mathrm{H3}$ (A) and NF-KB (P65) (B) in the nucleus after hsa_circ_0043278 overexpression in MDA-MB-231 cells.

Additional file 10: Figure S7. Western blot bands of $\beta$-actin (A) and NF-KB (P65) (B) in the cytosol after silencing hsa_circ_0043278 in MCF-7 cells.

Additional file 11: Figure S8. Western blot bands of histone $\mathrm{H3}$ (A) and NF-KB (P65) (B) in the nucleus after silencing hsa_circ_0043278 in MCF-7 cells.

Additional file 12: Figure S9. Western blot bands of EI24 (A) and $\beta$-actin (B) after hsa_circ_0043278 overexpression and/or transfection of the miR455-3p mimic in MDA-MB-231 cells.

Additional file 13: Figure S10. Western blot bands of $\beta$-actin (A) and El24 (B) after hsa_circ_0043278 downregulation and/or transfection of the miR455-3p inhibitor in MCF-7 cells.

Additional file 14: Figure S11. Western blot bands of NF-KB (P65) (A) and $\beta$-actin (B) in the cytosol after hsa_circ_0043278 overexpression and/or transfection of the miR-455-3p mimic in MDA-MB-231 cells.

Additional file 15: Figure S12. Western blot bands of histone $\mathrm{H3}$ (A) and NF-KB (P65) (B) in the nucleus after hsa_circ_0043278 overexpression and/ or transfection of the miR-455-3p mimic in MDA-MB-231 cells.

Additional file 16: Figure S13. Western blot bands of NF-KB (P65) (A) and $\beta$-actin (B) in the cytosol after hsa_circ_0043278 downregulation and/or transfection of the miR-455-3p inhibitor in MCF-7 cells.

Additional file 17: Figure S14. Western blot bands of histone $\mathrm{H3}$ (A) and NF-KB (P65) (B) in the nucleus after hsa_circ_0043278 downregulation and/or transfection of the miR-455-3p inhibitor in MCF-7 cells.

\section{Acknowledgements}

We want to thank the Department of Breast Surgery, The First Affiliated Hospital of China Medical University, for providing the BC tissues and related clinical data.

\section{Authors' contributions}

All authors participated in the conception and design of the study. CL conceived the project and supervised all experiments. YS conducted all experiments and analysed the data. $\mathrm{CL}$ and YS wrote the manuscript. All authors read and approved the final manuscript.

\section{Funding}

Not applicable.

\section{Availability of data and materials}

The datasets used during the current study are available from the corresponding author on reasonable request. 


\section{Declarations}

\section{Ethics approval and consent to participate}

The current study was approved by the Ethics Committee of The First Affiliated Hospital of China Medical University and performed in strict accordance with the Declaration of Helsinki. All participants signed informed consent forms prior to enrolment. All animal procedures were performed in accordance with the ARRIVE guidelines and the Guide for the Care and Use of Laboratory Animals published by the National Institutes of Health (NIH, Bethesda, Maryland USA). Great efforts were made to ensure minimal usage of animals as well as minimize their suffering.

\section{Consent for publication}

Not applicable.

\section{Competing interests}

The authors declare that they have no competing interests.

\section{Author details}

'Department of Geriatric Surgery, The First Affiliated Hospital of China Medical University, Shenyang 110001, China. ${ }^{2}$ Department of Breast Surgery, The First Affiliated Hospital of China Medical University, Shenyang 110001, China.

Received: 11 February 2021 Accepted: 10 November 2021

Published online: 20 November 2021

\section{References}

1. Parrella P. Epigenetic signatures in breast cancer: clinical perspective. Breast Care. 2010;5(2):66-73.

2. Siegel R, DeSantis C, Virgo K, Stein K, Mariotto A, Smith T, et al. Cancer treatment and survivorship statistics, 2012. CA Cancer J Clin. 2012;62(4):220-41.

3. Laessig D, Nagel D, Heinemann V, Untch M, Kahlert S, Bauerfeind I, et al. Importance of CEA and CA 15-3 during disease progression in metastatic breast cancer patients. Anticancer Res. 2007;27(4A):1963-8.

4. Stieber P, Nagel D, Blankenburg I, Heinemann V, Untch M, Bauerfeind I, et al. Diagnostic efficacy of CA 15-3 and CEA in the early detection of metastatic breast cancer-A retrospective analysis of kinetics on 743 breast cancer patients. Clin Chim Acta. 2015:448:228-31.

5. Zeng C, Guo X, Long J, Kuchenbaecker KB, Droit A, Michailidou K, et al. Identification of independent association signals and putative functional variants for breast cancer risk through fine-scale mapping of the 12p11 locus. Breast Cancer Res. 2016;18(1):64.

6. Zhang B, Beeghly-Fadiel A, Long J, Zheng W. Genetic variants associated with breast-cancer risk: comprehensive research synopsis, meta-analysis, and epidemiological evidence. Lancet Oncol. 2011;12(5):477-88.

7. Schnitt SJ. Classification and prognosis of invasive breast cancer: from morphology to molecular taxonomy. Modern Pathology. 2010;23(Suppl 2):S60-4.

8. Memczak S, Jens M, Elefsinioti A, Torti F, Krueger J, Rybak A, et al. Circular RNAs are a large class of animal RNAs with regulatory potency. Nature. 2013;495(7441):333-8.

9. Ebbesen KK, Kjems J, Hansen TB. Circular RNAs: identification, biogenesis and function. Biochim Biophys Acta. 2016;1859(1):163-8.

10. Li J, Sun D, Pu W, Wang J, Peng Y. Circular RNAs in cancer: biogenesis, function, and clinical significance. Trends Cancer. 2020;6(4):319-36.

11. Zheng $X$, Chen $L$, Zhou Y, Wang Q, Zheng Z, Xu B, et al. A novel protein encoded by a circular RNA circPPP1R12A promotes tumor pathogenesis and metastasis of colon cancer via Hippo-YAP signaling. Mol Cancer. 2019;18(1):47
12. Hansen TB, Jensen TI, Clausen BH, Bramsen JB, Finsen B, Damgaard CK, et al. Natural RNA circles function as efficient microRNA sponges. Nature. 2013:495(7441):384-8.

13. Meng X, Li X, Zhang P, Wang J, Zhou Y, Chen M. Circular RNA: an emerging key player in RNA world. Brief Bioinform. 2017;18(4):547-57.

14. Shao Y, Chen Y. Roles of circular RNAs in neurologic disease. Front Mo Neurosci. 2016:9:25.

15. Arnaiz E, Sole C, Manterola L, Iparraguirre L, Otaegui D, Lawrie CH. CircRNAs and cancer: biomarkers and master regulators. Semin Cancer Biol. 2018.

16. Zhang $X$, Wang S, Wang H, Cao J, Huang $X$, Chen Z, et al. Circular RNA circNRIP1 acts as a microRNA-149-5p sponge to promote gastric cancer progression via the AKT1/mTOR pathway. Mol Cancer. 2019;18(1):20.

17. Xu J, Chen X, Sun Y, Shi Y, Teng F, Lv M, et al. The regulation network and clinical significance of circular RNAs in breast cancer. Front Oncol. 2021:11:691317.

18. Chu M, Fang $Y$, Jin $Y$. CircRNAs as promising biomarker in diagnosis of breast cancer: an updated meta-analysis. J Clin Lab Anal. 2021:e23934

19. Liu C, Han T, Shi Y. The decreased expression of hsa_circ_0043278 and its relationship with clinicopathological features of breast cancer. Gland Surg. 2020;9(6):2044-53

20. Li Z, Meng Q, Pan A, Wu X, Cui J, Wang Y, et al. MicroRNA-455-3p promotes invasion and migration in triple negative breast cancer by targeting tumor suppressor El24. Oncotarget. 2017;8(12):19455-66.

21. Choi JM, Devkota S, Sung YH, Lee HW. El24 regulates epithelial-to-mesenchymal transition and tumor progression by suppressing TRAF2-mediated NF-kappaB activity. Oncotarget. 2013:4(12):2383-96.

22. Oeckinghaus A, Hayden MS, Ghosh S. at74e. Nat Immunol. 2011;12(8):695-708.

23. Oeckinghaus A, Hayden MS, Ghosh S. Crosstalk in NF-kappaB signaling pathways. Nat Immunol. 2011:12(8):695-708.

24. Bray F, Ferlay J, Soerjomataram I, Siegel RL, Torre LA, Jemal A. Global cancer statistics 2018: GLOBOCAN estimates of incidence and mortality worldwide for 36 cancers in 185 countries. CA Cancer J Clin. 2018;68(6):394-424.

25. Jeck WR, Sharpless NE. Detecting and characterizing circular RNAs. Nat Biotechnol. 2014;32(5):453-61.

26. Guo JU, Agarwal V, Guo H, Bartel DP. Expanded identification and characterization of mammalian circular RNAs. Genome Biol. 2014;15(7):409.

27. Ambros $V$. The functions of animal microRNAs. Nature. 2004;431(7006):350-5.

28. Bartel DP. MicroRNAs: target recognition and regulatory functions. Cell. 2009;136(2):215-33

29. Kloosterman WP, Plasterk $\mathrm{RH}$. The diverse functions of microRNAs in animal development and disease. Dev Cell. 2006;11(4):441-50.

30. Selbach M, Schwanhausser B, Thierfelder N, Fang Z, Khanin R, Rajewsky N. Widespread changes in protein synthesis induced by microRNAs. Nature. 2008;455(7209):58-63.

31. Shukla GC, Singh J, Barik S. MicroRNAs: processing, maturation, target recognition and regulatory functions. Mol Cell Pharmacol. 2011;3(3):83-92.

32. Salzman J, Gawad C, Wang PL, Lacayo N, Brown PO. Circular RNAs are the predominant transcript isoform from hundreds of human genes in diverse cell types. PLoS One. 2012;7(2):e30733.

33. Xu H, Guo S, Li W, Yu P. The circular RNA Cdr1as, via miR-7 and its targets, regulates insulin transcription and secretion in islet cells. Sci Rep. 2015:5:12453.

\section{Publisher's Note}

Springer Nature remains neutral with regard to jurisdictional claims in published maps and institutional affiliations. 